\title{
A REMARK TO THE NUMERICAL MODELLING OF 2D GEOSYNTHETIC TUBES
}

\section{JOZEF KAČUR, JOZEF MINÁR and JELA BABUŠÍKOVÁ}

Department of Physics

Radlinského 11, 81005

SvF STU Bratislava

Slovakia

e-mail: kacur@fmph.uniba.sk

Faculty of Mathematics, Physics and Informatics

Comenius University

Mlynska Dolina

84248 Bratislava

Slovakia

e-mail:minar@fmph.uniba.sk

babusikova@fmph.uniba.sk

\begin{abstract}
We discuss numerical modelling of 2D geosynthetic tubes on rigid and deformable foundation. Our mathematical model is a slight extension of previous ones in (Plaut and Suherman [11]) where the density of slurry material in tubes is constant. Now, density is a function linked to components of slurry material. Also, a simple and efficient numerical method is proposed endorsed by the series of experiments.
\end{abstract}

2010 Mathematics Subject Classification: 65K04, 65Z04, 86A04.

Keywords and phrases: geotubes, numerical modelling, model scaling.

Received January 25, 2017; Revised February 24, 2017

(ㄷ) 2017 Scientific Advances Publishers 


\section{Introduction}

Geosynthetic tubes have many applications in geotechnical engineering. These tubes are comprised of thin sheets and are filled with dragged slurry material in the form of tubes by pumping. They can be used to prevent beach erosion (Erchinger [3]), to prevent the spreading of pollution (Sprague and Fowler [13]), (Perrier [10]), flood protection (Recio and Oumerne [12]), (Huong et al. [4]), (Kim et al. [6]) etc. These tubes are long with the same cross-section, so they can be modelled as $2 \mathrm{D}$ objects. The shapes of geosynthetic tubes have been modelled and analyzed in many papers, e.g., (Kazimierowicz [5]; Leshchinsky et al. [7]; Plaut and Suherman [11]; Malik and Sysala [9]), etc. The synthetic sheet material is assumed as an inextensible membrane with negligible weight and the tube rest on a rigid, or deformable foundation. The rigid foundation has been considered in (Kazimierowicz [5]), (Leshchinsky et al. [7]), (Malik and Sysala [9]) while the deformable foundation in (Plaut and Suherman [11]). Floating and submerged tubes in water have been considered in (Zhao and Aarsnes [14]). Mathematical attributes as existence and uniqueness of the model solution have been discussed in (Antman and Schagerl [1]), (Malik [8]). Some numerical methods for the model solution are presented in (Cantre [2]). The density of the slurry material is assumed to be constant (an average was considered) except of (Malik and Sysala [9]), where slurry material is supposed to consists of $n(n \geq 1)$ different items with different densities. There is also presented an original numerical modelling which leads to a solution of nonlinear system of $4 n+5$ algebraic equations solved by Newton's method requiring a good starting point. Some model parameters in the algebraic system require evaluation of some integrals. The obtained numerical results are in good agreement with the previous results (for $n=1$ ), which were also compared (in special cases) to semi-analytical solutions. 
In our contribution, we consider a slight model extended by a general density corresponding to the slurry material with arbitrary items (e.g., $n=\infty$ ). We also propose a simple and efficient numerical method which does not depend on the number of slurry items and could be easily realized in MATLAB software. The method is based on solving 5 (for rigid foundation) and 6 (for deformable foundation) ODE equations combined with a function minimization that has three parameters. A simple iterative procedure also requires a good starting point, but we present original numerical approach which leads to the solution for the most of starting points.

In Section 2, we present a mathematical model, which is a slight extension of that ones in (Plaut and Suherman [11]) for the rigid foundation. In Section 3, the deformable foundation is considered. The proposed numerical method is discussed in Section 4. Presentation of a numerical procedure with many numerical experiments is given in Section 5.

\section{Geosynthetic Tube on Rigid Foundation}

A cross-section of the tube is sketched in Figure 1. We consider the consolidated state of slurry in the tube, where the density $\gamma(y)$ of slurry particles is non-increasing function in the vertical variable $y, 0 \leq y \leq H$ ( $y=0$ corresponds to the rigid foundation), where $H$ is a priori not known and underly to the model solution. The tube sheet (membrane) has the prescribed perimeter $L$ so that the maximal cross-section area of

the geosynthetic tube is $V_{\max }=\frac{L^{2}}{4 \pi}$. We assume that the amount of slurry material corresponds to the cross-sectional area $V_{z}\left(0 \leq V_{z}<\right.$ $\left.V_{\max }\right)$. The cross-section boundary in contact with the rigid foundation is flat, $y=0$ for $-s_{0} \leq x \leq s_{0}$, where $x$ is the horizontal variable. The shape of the cross-sectional area, pressure on the top and bottom of the tube, length of the contact zone, and height of the tube are the primary 
unknowns that have to be determined. Due to the symmetry of the crosssectional area, it is sufficient to consider only half of this area where $0 \leq x \leq s_{0}, y \in(0, H)$. Here $s_{0}$ is also a priori not known and is part of the model solution. As suggested in (Plaut and Suherman [11]), it is very suitable to parametrize the boundary of considered area using parameter $s$ representing the arch length from the point $(x(s), y(s))$ to the origin $(0,0)$ on the boundary. Then, for $s \geq s_{0}$, we move upwards up to the parameter value $s=L / 2$. So the boundary is expressed by $(x(s), y(s))$ and for $s \geq s_{0}$ we have a unique correspondence between $s$ and $y(s)$ ( $y$ is strictly increasing in $s$ ).

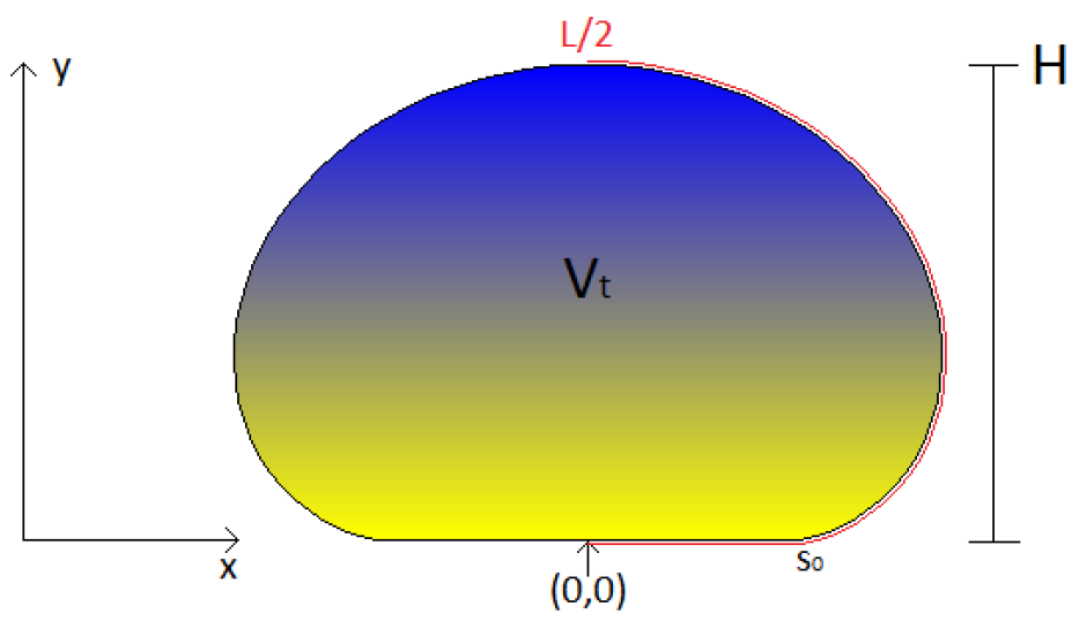

Figure 1. Cross-section diagram for geosynthetic tube.

To modify the governing system in (Plaut and Suherman [11]), (Malik and Sysala [9]) for variable density we require the density characterization of the used slurry by a density distribution function $R o(V)$ which can be defined as follows. Consider slurry which has volume $V_{z}$ of the cross-sectional area. We consolidate this volume of slurry in a fixed cylinder which is scaled in terms of volume from bottom $V=0$ up to the top $V=V_{z}$. 
Definition 1. The density distribution function $R o(V)$ represents the density of consolidated slurry at the level $V$ in the considered cylinder. The smaller the cross-section of the cylinder, the more precise scaling of $R o(V)$. Finally, let $\theta \equiv \theta(s)$ represent the angle between the horizontal axis and the tangent to the tube at the boundary point $(x(s), y(s))$. Then the governing mathematical model consists of the following ODE for $0<s \leq s_{0}$ :

$$
\begin{gathered}
\partial_{s} x(s)=\cos (\theta(s)), \quad x\left(s_{0}\right)=s_{0}, \\
\partial_{s} y(s)=\sin (\theta(s)), \quad y\left(s_{0}\right)=0, \\
\partial_{s} V(s)=2 x(s) \sin (\theta(s)), \quad V\left(s_{0}\right)=0, \\
\partial_{s} p(s)=-g R o(V(s)) \sin (\theta(s)), \quad p\left(s_{0}\right)=p_{0}, \\
t \partial_{s} \theta(s)=p(s), \quad \theta\left(s_{0}\right)=0,
\end{gathered}
$$

with additional conditions: $\quad x(L / 2)=0, \theta(L / 2)=\pi, V(L / 2)=V_{z}$, where $p_{0}, t, s_{0}$ are unknown. This system is a slight extension of that ones in (Plaut and Suherman [11]), (Malik and Sysala [9]). So we have 3 unknowns which should be determined in such a way that 3 conditions will be satisfied under the validity of previous 5 ODE. Here, variable $V(s)$ represents the amount of slurry contained in the area from bottom up to the height $y(s), p(s)([\mathrm{Pa}])$ represents pressure in the tube in the height $y(s)$ and $t([\mathrm{~N} / \mathrm{m}])$ represents the tension in the synthetic fabric, which is constant in our setting.

\section{Geosynthetic Tube on Deformable Foundation}

The deformable foundation is supposed to be of WINKLER type where the resistance of the foundation is proportional to its deformation. It is similar to a resistance of loaded spring. Now, this model reads as follows: 


$$
\begin{gathered}
\partial_{s} x(s)=\cos (\theta(s)), \quad x(0)=0 \\
\partial_{s} y(s)=\sin (\theta(s)), \quad y(0)=-h_{f} \\
\partial_{s} V(s)=2 x(s) \sin (\theta(s)), \quad V(0)=0 \\
\partial_{s} p(s)=g D(V(s))\left\{\alpha K_{f}\left[\cos (\theta(s))-\frac{p(s)}{t} y(s)\right]-1\right\} \sin (\theta(s))=0, p(0)=p_{0} \\
t \partial_{s} \theta(s)=p(s), \quad \theta(0)=0, \\
\partial_{s} t(s)=\alpha K_{f} g R o(V(s)) y(s) \sin (\theta(s)), \quad t(0)=t_{0}
\end{gathered}
$$

with

$$
\alpha \equiv \alpha(y)=1 \text {, if } y<0, \alpha(y)=0 \text {, if } y \geq 0 .
$$

Our mathematical model is the same as that one in (Plaut and Suherman [11]), except to the non-constant density as stated in our model in Section 2. Here, $K_{f}$ is the stiffness coefficient (non-dimensional) of the deformable foundation, $h_{f}$ is the depth of the bottom of the tube with respect to the surface of the undeformed foundation. Here, we have also 3 unknown model parameters $P=\left\{h_{f}, p_{0}, t_{0}\right\}$ by means of which (under assumption of (6)-(11)), we have to satisfy the following conditions:

$$
x(L / 2)=0, \quad \theta(L / 2)=\pi, \quad V(L / 2)=V_{z} .
$$

Remark 1. The mathematical model of submerged tubes in the water is similar to the model for deformable foundation, where in WINKLER model a hydrostatic force is applied in the vertical direction.

\section{Proposed Numerical Method}

In order to use a professional ODE solver to solve either of the systems, the unknown parameters $P_{n d}=\left\{p_{0}, s_{0}, t\right\}$ for the problem with 
rigid foundation and $P_{d}=\left\{p_{0}, h_{f}, t_{0}\right\}$ for the problem with deformable foundation have to be computed. The natural numerical method to solve the problem is to satisfy the required conditions in an iterative way.

\subsection{Simple minimization method}

The most simple approach is to solve the minimization problem:

$$
\min _{P} J(P)=x_{P}^{2}(L / 2)+\left(\theta_{P}(L / 2)-\pi\right)^{2}+\left(V_{P}(L / 2)-V_{z}\right)^{2},
$$

where $x_{P}, \theta_{P}, V_{P}$ are the solution of (1)-(5) (for deformable foundation system (6)-(11)) corresponding to the vector parameter $P$. Solution of (12) can be easily obtained by the solver "fminsearch" (Nelder-Mead method), while the solution of ODE system with a vector parameter $P$ can be found by the solver "ode45" (Dormand Prince method) from MATLAB library. In an iterative way, the functional is minimized and this process finishes when $J\left(P_{o p t}\right)<$ Tolerance. In this way, it is sufficient to input starting parameter $P_{\text {start }}$ into the corresponding code and we obtain the required solution $P_{o p t}$. Then we analyze whether the obtained solution $P_{o p t}$ is the desired solution or a local minimum (in which case we have to restart the minimization with the better starting point).

\subsection{Successive subproblems minimization method}

We have also developed a slower, yet more reliable numerical approach. It consists of four stages, which gradually improves initial guess.

In the first step, we run the ODE system (using ode45) for some starting point $P_{0}$ and obtain solution of the ODE. We distinguish between the case of rigid foundation and the case of deformable foundation. In the case of deformable foundation:

- If $p(s)<0$ for any $s \in(0, L / 2)$, then we set $p_{0}:=2 p_{0}$.

- If $t(s)<0$ or $\theta(s)<0$ or $\theta(s)>\frac{3}{2} \pi$ for any $s \in(0, L / 2)$, then we set $t_{0}:=2 t_{0}$. 
- If we changed starting point, we reset Stage 1 , otherwise we continue to Stage 2 .

In the case of rigid foundation:

- If $p(s)<0$ for any $s \in(0, L / 2)$, then we set $p_{0}:=2 p_{0}$.

- If $\theta(s)<0$ or $\theta(s)>\frac{3}{2} \pi$ for any $s \in(0, L / 2)$, then we set $t:=2 t$.

- If we changed starting point, we reset Stage 1 , otherwise we continue to Stage 2 .

In Stage 2, we use starting point $P$ obtained in Stage 1 to run the following minimization problem (using fminsearch):

$$
\min _{P} J(P)=\left(\theta_{P}(L / 2)-\pi\right)^{2} .
$$

In Stage 3, we use starting point $P$ obtained in Stage 2 to run the following minimization problem (using fminsearch):

$\min _{P} J(P)= \begin{cases}{\left[c_{3}^{2}-\left(\theta_{P}(L / 2)-\pi\right)^{2}\right]^{-1}+K_{3} x_{P}(L / 2)^{2},} & \text { if }\left|\theta_{P}(L / 2)-\pi\right|<c_{3}, \\ \infty, & \text { otherwise },\end{cases}$

where $c_{3}=\pi / 100$ and $K_{3}=1000$.

Finally, in Stage 4, we use starting point $P$ obtained in Stage 3 to run the following minimization problem (using fminsearch):

$$
\begin{aligned}
& \min _{P} J(P)= \\
& \begin{cases}{\left[c_{4}^{2}-\left(x_{P}(L / 2)\right)^{2}\right]^{-1}} & \text { if }\left|\theta_{P}(L / 2)-\pi\right|<c_{3} \\
+\left[c_{3}^{2}-\left(\theta_{P}(L / 2)-\pi\right)^{2}\right]^{-1}+K_{4}\left(V_{P}(L / 2)-V_{z}\right)^{2}, & \text { and }\left|x_{P}(L / 2)\right|<c_{4}, \\
\infty, & \text { otherwise, }\end{cases}
\end{aligned}
$$

where $c_{4}=L / 500$ and $K_{4}=1000$. 
At the end of Stage 4, we compute desired values $P$. We note that this minimization method is more time consuming than simple minimization method from Subsection 4.1 and the relative and absolute tolerances in the MATLAB ode solver have to be set sufficiently low. However, it converges to the desired solution for most starting points (unlike simple minimization method, which converges to a local minimum more often than not).

\section{Numerical Experiments}

In this section, we demonstrate the numerical process of successive subproblems minimization method and present some shapes of geosynthetic tubes for given parameters and densities.

\subsection{Demonstration of successive subproblems minimization method for rigid foundation}

We demonstrate the process of successive subproblems minimization method in Figure 2. We plot the curve $(x(s), y(s))$ using a full line and the curve $(-x(s), y(s))$ representing the symmetric part of the tube using a dotted line in each graph, where $0<s<L / 2$. We have used the following input:

$$
V=6, \quad L=10, \quad g=9.81, \quad R o(V)= \begin{cases}1300 & \text { if } V<1.5, \\ 1200 & \text { if } 1.5 \leq V<3, \\ 1100 & \text { if } 3 \leq V<4.5, \\ 1000 & \text { if } 4.5 \leq V .\end{cases}
$$




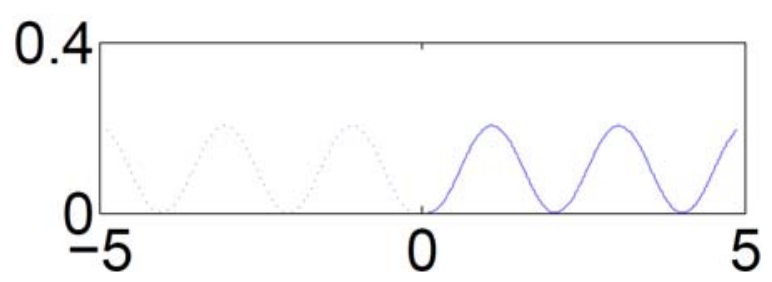

(a) Before phase 1: $s_{0}=0.1, p_{0}=1000, t=1000$.

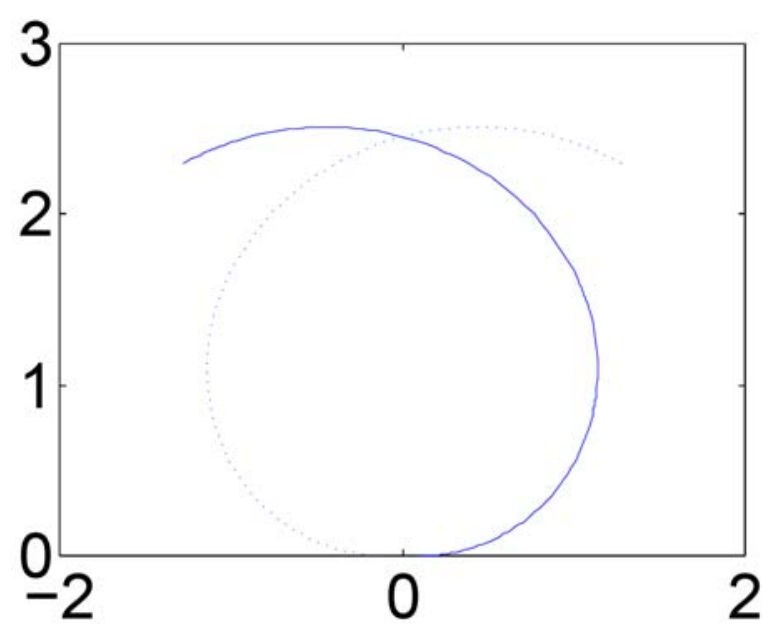

(b) After phase $1: s_{0}=0.1, p_{0}=64000, t=64000$.

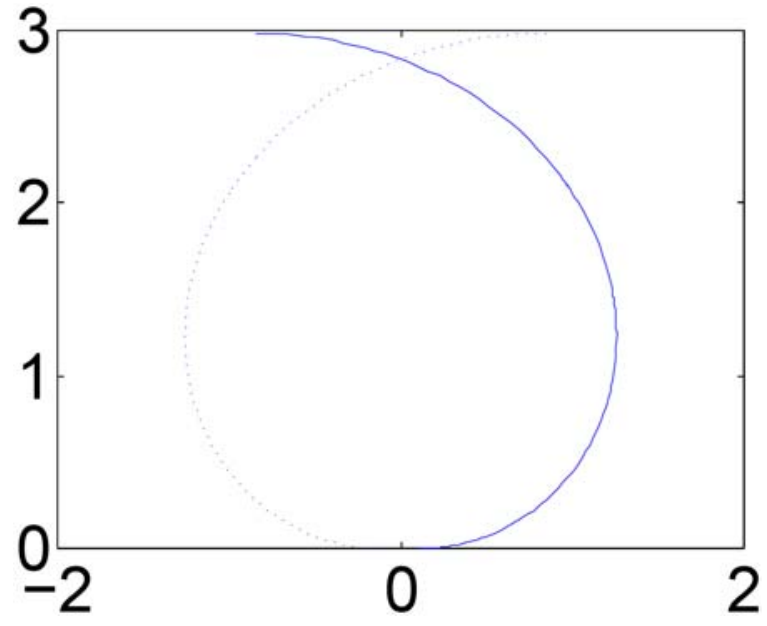

(c) After phase $2: s_{0}=0.104, p_{0}=61015, t=67243$. 


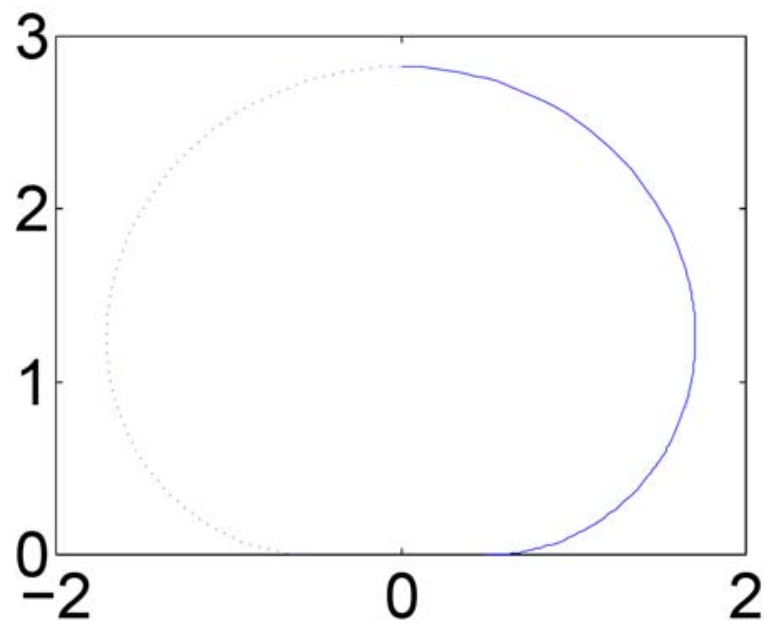

(d) After phase $3: s_{0}=0.498, p_{0}=91283, t=106900$.

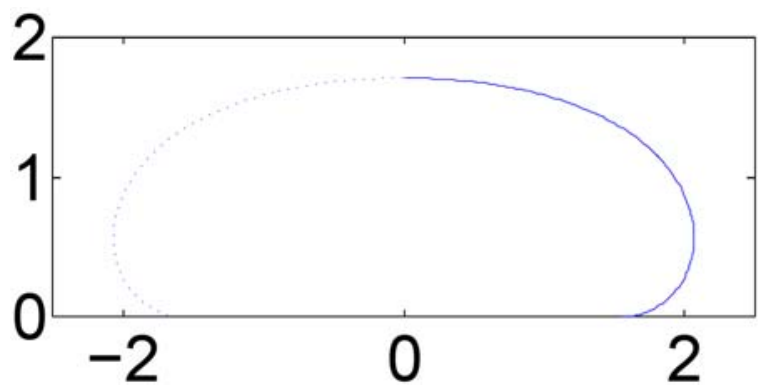

(e) After phase $4: s_{0}=1.548, p_{0}=21860, t=10786$.

Figure 2. Demonstration of successive subproblems minimization method for the rigid foundation.

Remark 2. We note that to obtain the correct vector parameter $P_{\text {opt }}=\left\{p_{0}=21951, s_{0}=1.548, t=10836\right\} \quad$ we have started with $P_{\text {start }}=\{1000,0.1,1000\}$ which demonstrates the effeciency of our algorithm. 
Remark 3. As we can see in our pictures, in Stage 1, we exclude unacceptable parameters $p$ and $t$. From admissible parameters in Stage 1 , we prepare new parameters where the validity of angle condition is satisfied. Then in Stage 3, we focus on connection of tube boundary keeping acceptable values of the angle. Finally, in Stage 4, we force validity of the volume condition, while keeping both angle and connection conditions in tolerance.

\subsection{Demonstration of successive subproblems minimization method for deformable foundation}

We demonstrate the process of successive subproblems minimization method in Figure 3. Again, we plot the curve $(x(s), y(s))$ using a full line and the curve $(-x(s), y(s))$ representing the symmetric part of the tube using dotted line in each graph, where $0<s<L / 2$. The black segment in each graph represents undeformed surface level. We have used the input:

$$
V=12, \quad L=15, \quad g=9.81, \quad K_{f}=10, \quad R o(V)=1350-10 V-V^{2} .
$$




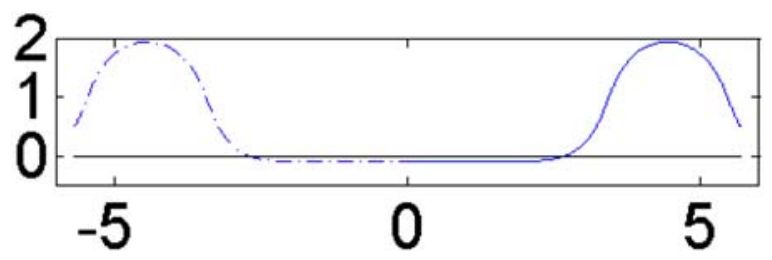

(a) Before phase 1: $h_{f}=0.1, p_{0}=2, t_{0}=10000$.

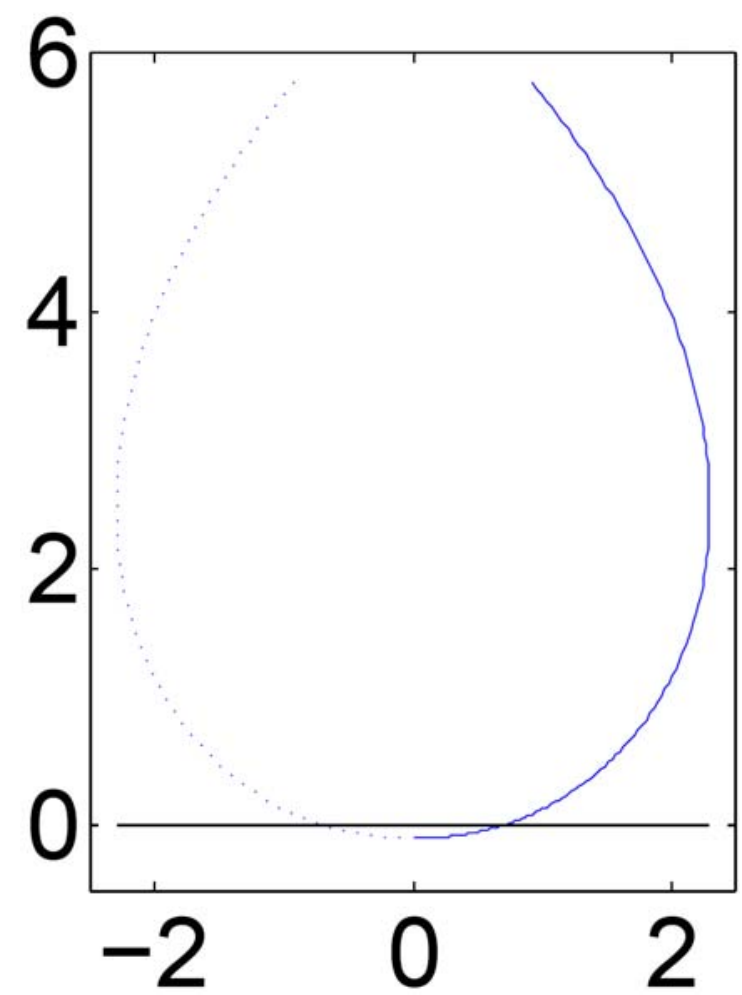

(b) After phase $1: h_{f}=0.1, p_{0}=65536, t_{0}=160000$. 


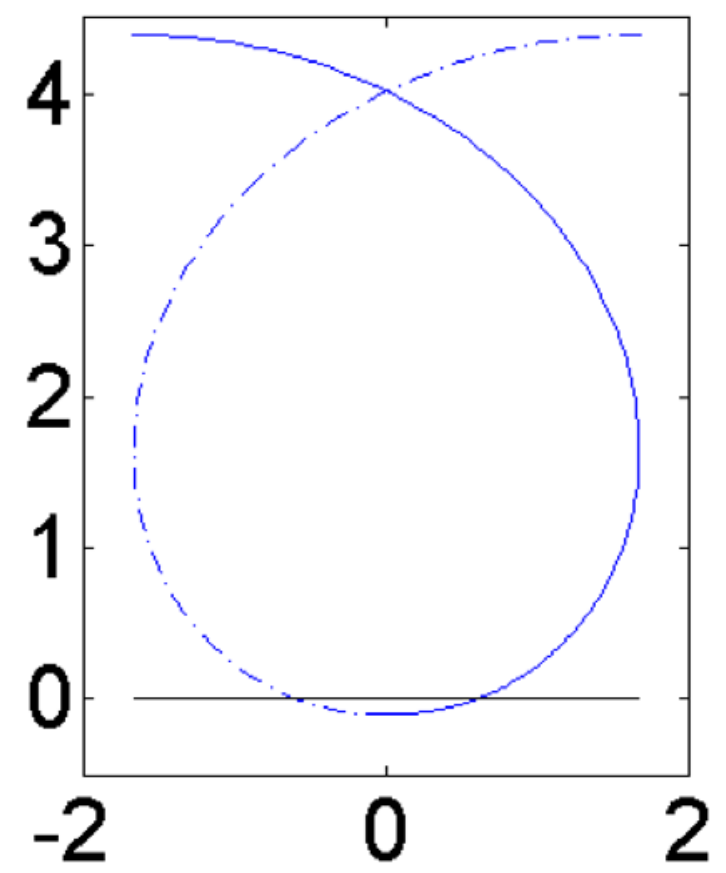

(c) After phase $2: h_{f}=0.105, p_{0}=74037, t_{0}=134200$.

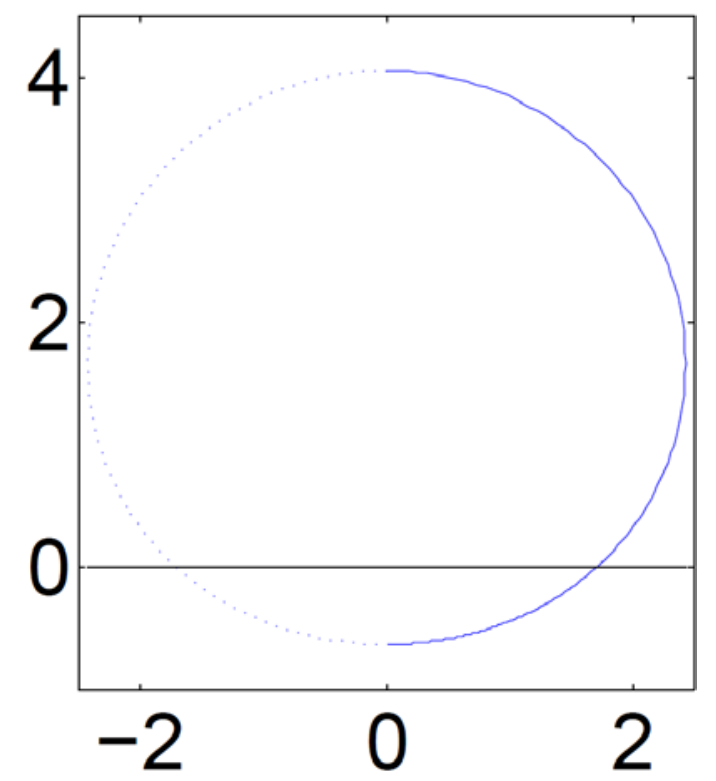

(d) After phase $3: h_{f}=0.622, p_{0}=308670, t_{0}=858690$. 


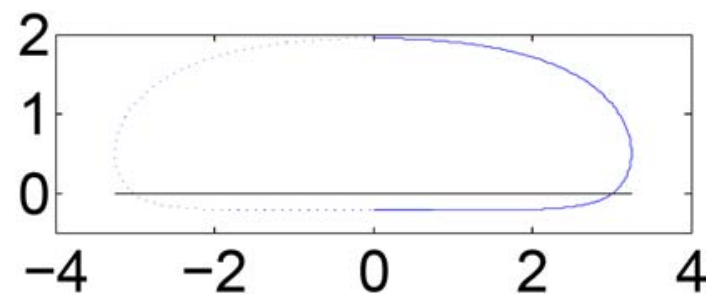

(e) After phase $4: h_{f}=0.207, p_{0}=11.874, t_{0}=16241$.

Figure 3. Demonstration of successive subproblems minimization method for the deformable foundation.

The effect of the algorithm is similar to the rigid foundation.

\subsection{Demonstration of some results for rigid foundation highlighting influence of various parameters}

In Figure 4, we demonstrate some final results for various input parameters. Under each graph, we present input parameters and computed output parameters. Quite naturally, increasing the perimeter of the tube yields more eccentric shape and lower pressure and tension. Conversely, the density function has very little influence on the final shape. The pressure and tension tend to increase when we increase the density. 


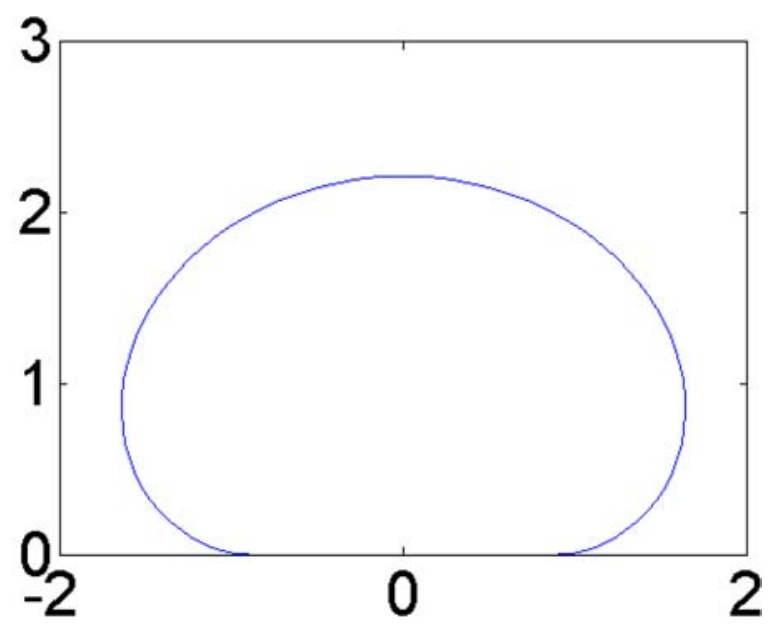

(a) Input parameters: $V=6, L=9, R o(V)=1500-125 \mathrm{~V}$.

Output parameters: $s_{0}=0.8661, p_{0}=3.8227 .10^{4}$, $t=2.7528 .10^{4}$.

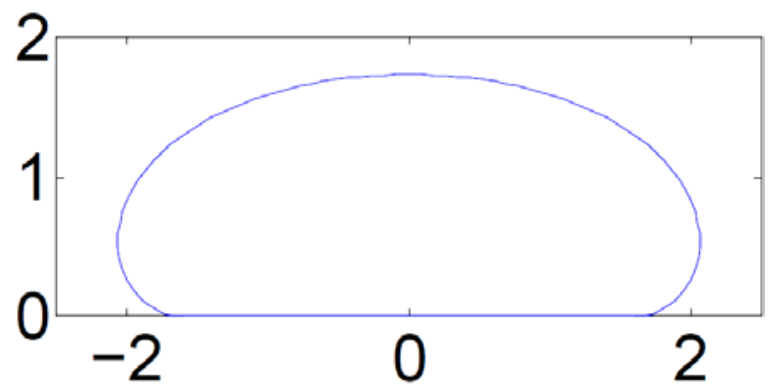

(b) Input parameters: $V=6, L=10, R o(V)=1500-125 V$.

Output parameters: $s_{0}=1.5968, p_{0}=2.0735 .10^{4}$, $t=8.9876 .10^{3}$. 


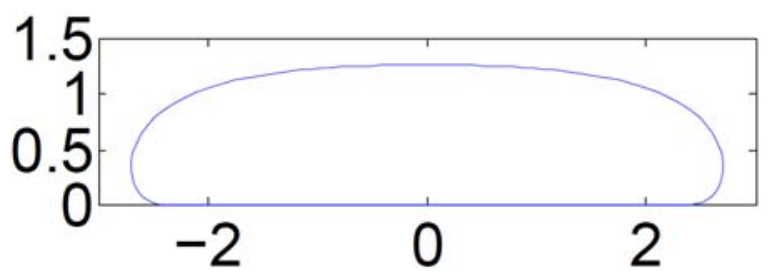

(c) Input parameters: $V=6, L=12, R o(V)=1500-125 V$.

Output parameters: $s_{0}=2.4, p_{0}=1.3795 .10^{4}$, $t=3.9231 .10^{3}$.

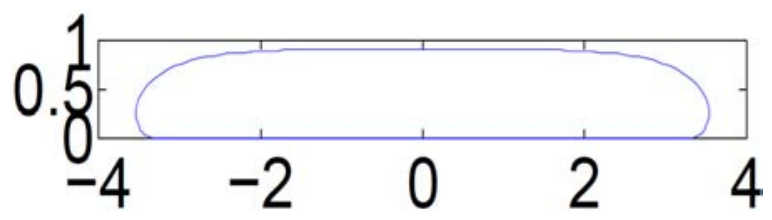

(d) Input parameters: $V=6, L=15, R o(V)=1500-125 V$.

Output parameters: $s_{0}=3.3236, p_{0}=9.9559 .10^{3}$, $t=2.0198 .10^{3}$.

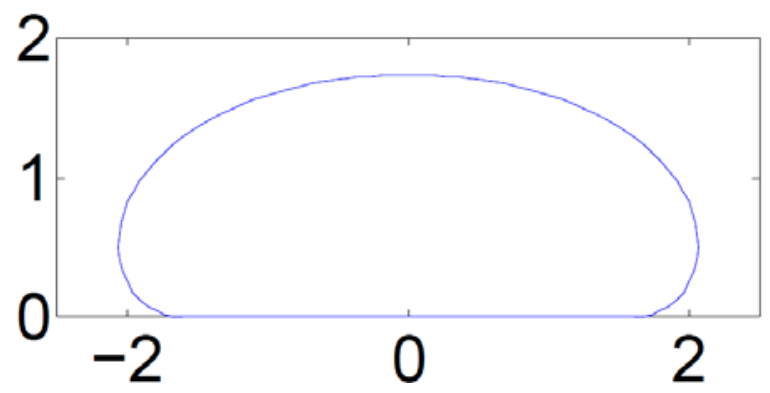

(e) Input parameters: $V=6, L=10, R o(V)=1500-150 V$.

Output parameters: $s_{0}=1.6069, p_{0}=1.9231 .10^{4}$, $t=8.1069 .10^{3}$. 


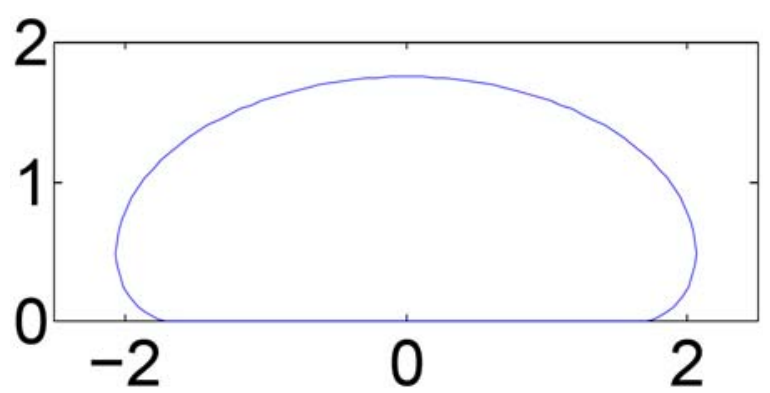

(f) Input parameters: $V=6, L=10, R o(V)=1500-200 V$.

Output parameters: $s_{0}=1.6334, p_{0}=1.6216 .10^{4}$, $t=6.3453 .10^{3}$.

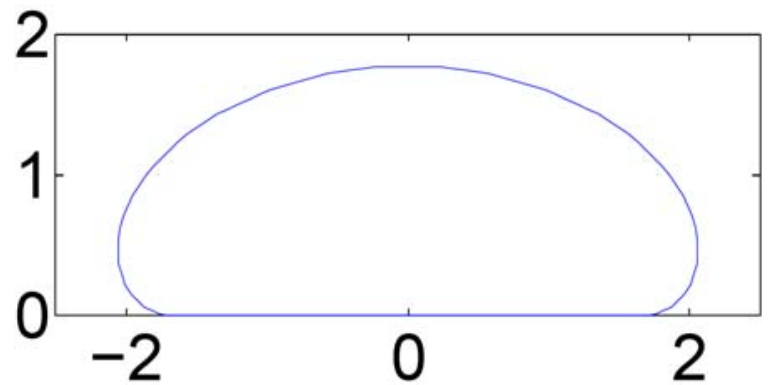

(g) Input parameters: $V=6, L=10, R o(V)=1500-230 \mathrm{~V}$.

Output parameters: $s_{0}=1.6552, p_{0}=1.4402 .10^{4}$, $t=5.2893 .10^{3}$. 


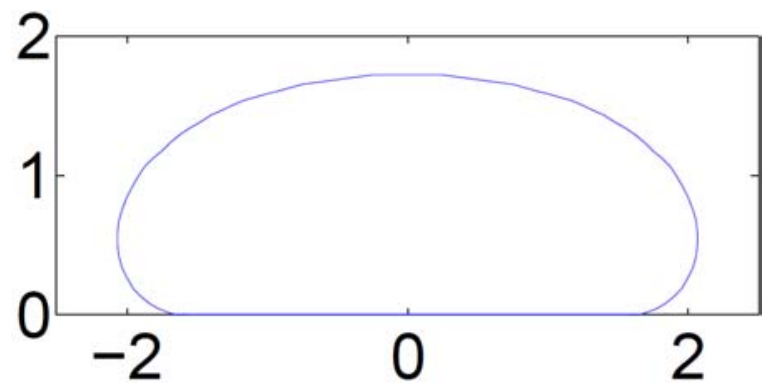

(h) Input parameters: $V=6, L=10, R o(V)=1500-2^{1.5 V}$.

Output parameters: $s_{0}=1.5776, p_{0}=2.6455 .10^{4}$, $t=1.2163 .10^{3}$.

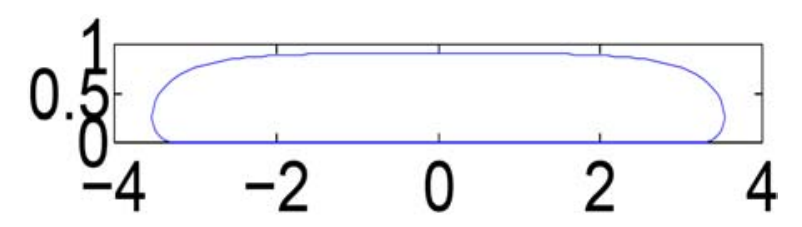

(i) Input parameters: $V=6, L=15, R o(V)=1500-2^{1.5 V}$.

Output parameters: $s_{0}=3.3083, p_{0}=1.2610 .10^{4}$, $t=2.7594 .10^{3}$.

Figure 4. Various results for rigid foundation.

\subsection{Demonstration of some results for deformable foundation highlighting influence of various parameters}

In Figure 5, we demonstrate some final results for various input parameters. Under each graph, we present input parameters and computed output parameters. Since we have done similar comparison in previous subsection, we focus mainly on stiffness coefficient $K_{f}$. We can see that for large values of $K_{f}$, the resulting shape is identical to shape obtained using the rigid foundation model with equal input parameters. 
Lower stiffness coefficients lead to the deeper lay-down and more curved bottom of the tube. While the change in $K_{f}$ affects only slightly the tension $t_{0}$ in the bottom of the tube, it has a significant impact on the bottom pressure $p_{0}$, which can be best seen in graphs (d)-(f).

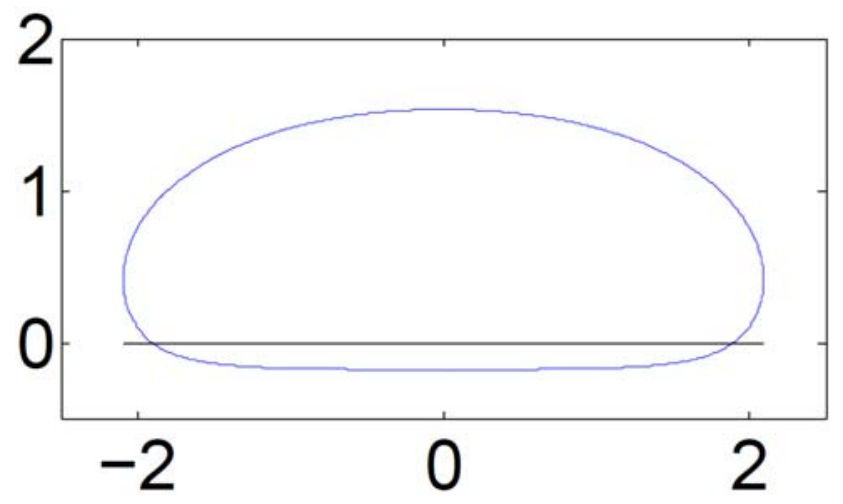

(a) Input parameters: $V=6, L=10, R o(V)=1500-2^{1.5 V}, K_{f}=10$.

Output parameters: $h_{f}=0.1734, p_{0}=78.2558, t_{0}=1.2487 .10^{4}$.

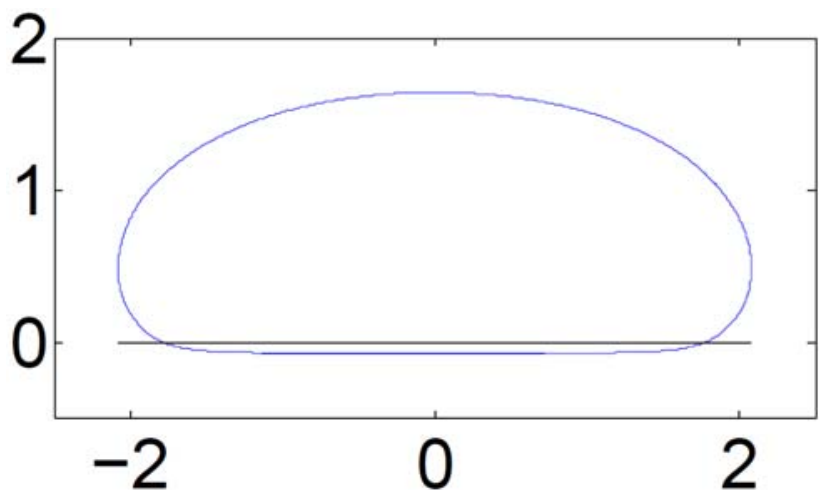

(b) Input parameters: $V=6, L=10, R o(V)=1500-2^{1.5 V}, K_{f}=25$.

Output parameters: $h_{f}=0.0708, p_{0}=3.2510, t_{0}=1.2241 .10^{4}$. 


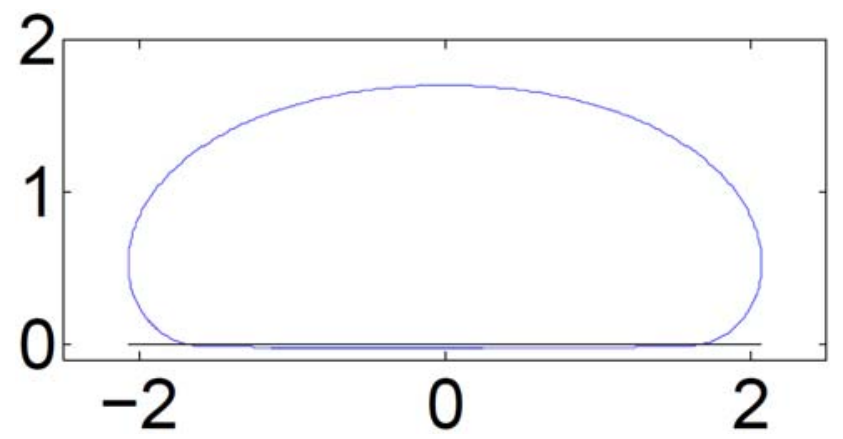

(c) Input parameters: $V=6, L=10, R o(V)=1500-2^{1.5 V}, K_{f}=100$.

Output parameters: $h_{f}=0.0179, p_{0}=5.6286 .10^{-4}, t_{0}=1.217 .10^{4}$.

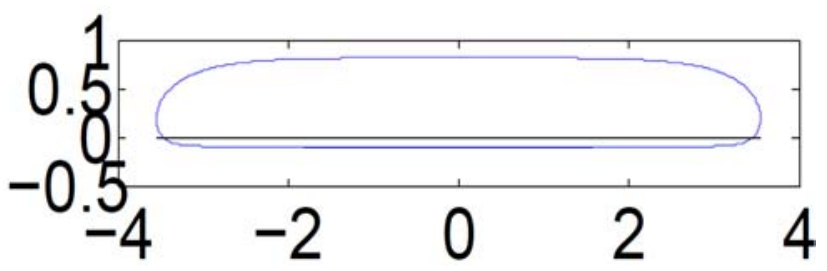

(d) Input parameters: $V=6, L=15, R o(V)=1500-2^{1.5 V}, K_{f}=10$.

Output parameters: $h_{f}=0.0854, p_{0}=1.9794 .10^{-6}, t_{0}=2.9974 .10^{3}$.

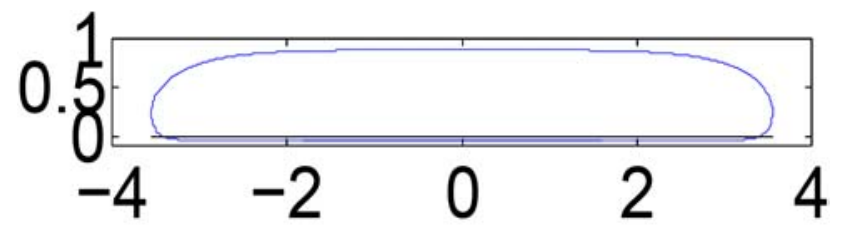

(e) Input parameters: $V=6, L=15, R o(V)=1500-2^{1.5 V}, K_{f}=25$.

Output parameters: $h_{f}=0.0342, p_{0}=8.3208 .10^{-13}, t_{0}=2.8525 .10^{3}$. 


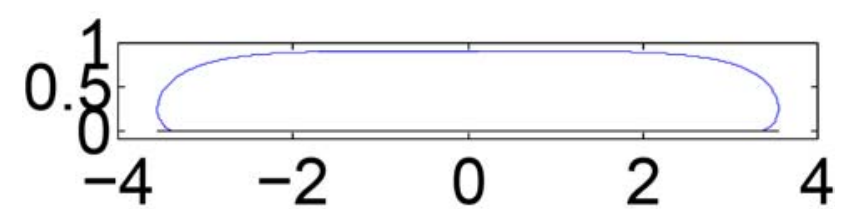

(f) Input parameters: $V=6, L=15, R o(V)=1500-2^{1.5 V}, K_{f}=100$.

Output parameters: $h_{f}=0.008575, p_{0}=1.1882 .10^{-29}$, $t_{0}=2.7816 .10^{3}$.

Figure 5. Various results for deformable foundation.

\section{Conclusion}

We present two mathematical models for $2 \mathrm{D}$ geosynthetic tubes modelling - one for the rigid foundation and one for the deformable foundation. These models are natural extension of previously published models, where we consider non-constant density in terms of an arbitrary non-increasing function. The numerical method is proposed in two versions. The first one is a very simple, but requiring suitable starting vector of unknown model parameters. The second one consist of multiple application of the first numerical model, where the cost functional is changed in a suitable way. In this case, we successively improve the starting point up to the final solution. The efficiency of the proposed algorithm is demonstrated in numerical experiments. The second version converges to the solution even for extreme initial guesses of the required model vector, where the first version would fail. However, quite naturally, if the first approach converges then it is faster than the second approach. We have presented the sequence of steps of the second approach on two examples. We have also presented many results for both rigid and deformable foundations and discussed the influence of various parameters. In this article, we have omitted to give a direct comparison of our results to the results listed in previous articles for reasons of 
abundance. However, we have compared our numerical results to that in

(Malik and Sysala [9]) and they coincide in very high degree (to all decimal points published in (Malik and Sysala [9]), Table 1).

\section{Acknowledgements}

The authors conform support by the Slovak Research and Development Agency APVV-15-0681 and VEGA 1/0565/16.

\section{References}

[1] S. Antman and M. Schagerl, Slumping instabilities of elastic membranes holding liquids and gases, International Journal of Non-linear Mechanics 40 (2005), 1112-1138.

[2] S. Cantre, Geosynthetic tubes/analytical design aspect, Geotextiles and Geomembranes 20 (2002), 305-319.

[3] H. Erchinger, Geotextiele Tubes Filled with Sand for Beach Erosion Control, North Sea Coast, Germany, Volume 1, BiTech Publishers, Richmond, BC, 1994.

[4] T. Huong, R. Plaut and G. Fila, Wedget geomembrane tubes as temporary floodfighting devices, Thin-Walled Structures 40 (2002), 913-923.

[5] K. Kazimierowicz, Simple Analysis of Deformation of Sand-sausages, Volume 2, Hydraulic Applications and Related Research, Singapore, 1994.

[6] M. Kim, M. Freeman, B. FitzPatrick, D. Nevius, R. Plaut and G. Filz, Use of an apron to stabilize geomembrane tubes for fighting floods, Geotextiles and Geomembranes 22 (2004), 239-254.

[7] D. Leshchinsky, O. Leshchinsky, H. Ling and P. Gilbert, Geosynthetic tubes for confining pressurized slurry: Some design aspects, Journal of Geotechnical Engineering 122 (1996), 682-690.

[8] J. Malik, Some problems connected with 2D modeling of geosynthetic tubes, Nonlinear Analysis: Real World Applications 10 (2009), 810-823.

[9] J. Malik and S. Sysala, Analysis of geosynthetic tubes filled with several liquids with different densities, Geotextiles and Geomembranes 29 (2011), 249-256.

[10] H. Perrier, Use of Soil-filled Synthetic Pillows for Erosion Protection, Volume 1, Vienna, 1986.

[11] R. Plaut and S. Suherman, Two-dimensional analysis of geosynthetic tubes, Acta Mechanica 129 (1998), 207-218. 
[12] J. Recio and H. Oumerne, Hydraulic permeability of structures made of geotextile sand containers: Laboratory test and conceptual model, Geotextiles and Geomembrans 26 (2008), 473-487.

[13] C. Sprague and J. Fowler, Dredged material-filled geotextile containers: Case histories, research and upcomming workshop, Industrial Fabrics Association International 1 (1994), 42-54.

[14] R. Zhao and J. Aarsnes, Numerical and experimental studies of floating and liquidfilled membrane structure in waves, Ocean Engineering 25 (1998), 753-765. 